\title{
Skin Infections in Indiana Nursing Homes
}

\author{
Kayla Brown ${ }^{1}$, Kathleen Unroe, MD, MHA ${ }^{1,2,3}$, Jennifer Carnahan, MD, MPH, \\ $\mathrm{MA}^{1,2,3}$ \\ ${ }^{1}$ Indiana University School of Medicine, ${ }^{2}$ Regenstrief Institute, Inc., ${ }^{3}$ Indiana \\ University Center for Aging Research
}

\begin{abstract}
Background and Hypothesis:
OPTIMISTIC (Optimizing Patient Transfers, Impacting Medical Quality, \& Improving Symptoms: Transforming Institutional Care) is a CMS (Centers for Medicare and Medicaid Services) demonstration project that aims to improve nursing home care. CMS provides enhanced reimbursement to nursing homes in the project when they are able to treat six conditions in the facility rather than being transferred to a hospital, one of which being skin infections. The purpose of this study is to examine the diagnosis, treatments, and outcomes of skin infections of residents in nursing homes enrolled in OPTIMISTIC.
\end{abstract}

\section{Experimental Design or Project Methods:}

A chart review of 26 cases was conducted using data from nursing home medical records of nine OPTIMISTIC facilities. Cases were randomly selected from billing episodes between November 2018 and May 2019. Data was entered into an Excel extraction tool created for the project and analyzed.

\section{Results:}

Of the randomly selected skin infections, $46.2 \%$ were diagnosed as non-purulent cellulitis and $23.1 \%$ as purulent cellulitis. Other infections included abscesses, infected pressure injuries, and shingles. Doxycycline was the most common antibiotic prescribed, used in $42.3 \%$ of the total skin infections. For non-purulent cellulitis, cephalexin was the next most used antibiotic (41.7\%). Twelve of the skin infections were recurrent, and the most common antibiotic used for those infections was doxycycline. Of the twenty-six cases, twenty-three were resolved in the facility, one was recertified for a longer length of time, one was transferred to the hospital, and one was transferred to hospice.

\section{Conclusion and Potential Impact:}

This project provides a unique opportunity to examine diagnosis and treatment patterns of skin infections in nursing homes. The results of this study will be used to bolster and augment future efforts at nursing home care improvement for patients diagnosed with skin infections in the facility. 